\title{
Circulating and Storage Neutrophil Changes in Experimental Type II Group B Streptococcal Sepsis
}

\author{
ROBERT D. CHRISTENSEN, ${ }^{\text {ron }}$ ANN O. SHIGEOKA, HARRY R. HILL, AND (iERALD ROTHSTEIN \\ Howard Hughes Medical Institute Laboratories. Departments of Medicine. Pediatrics, and Pathologi. Primary \\ Children's Medical Center, and the University of Utah College of Medicine. Salt Lake (ity. Utah. USA
}

\begin{abstract}
Summary
The availibility of neutrophils is an important factor in host resistance to bacterial infection. Therefore, circulating and storage neutrophil quantification was carried out on groups of neonatal rats intranasally inoculated with type II group B streptococci. The dose of type II group B streptococci used produced $56 \%$ mortality in 48 neonatal rats with death being due to pneumonia and sepsis. Neutropenia $\left(1300 \pm 150 / \mathrm{mm}^{3}\right.$ versus $2300 \pm 170 / \mathrm{mm}^{3}$; mean \pm S.D.: $P<0.01$ ) and an elevation in band/polymorphonuclear ratio $(0.98 \pm 0.04$ versus $0.30 \pm 0.04 ; P<0.01)$ were observed in infected neonatal rats $24 \mathrm{hr}$ following inoculation. Femoral marrow as well as splenic and hepatic neutrophil storage compartment quantification revealed dimunition of postmitotic (polymorphonuclear, band, and metamyelocyte) neutrophils in the infected group $(P<$ 0.01 ) with sparing of the proliferative neutrophils (myeloblasts, promyelocytes, and myelocytes). Repletion of the myeloid but not the splenic or hepatic neutrophil storage compartments with normalization of the neutrophil count and band/polymorphonuclear ratio occurred in animals surviving $72 \mathrm{hr}$. These studies establish that neutropenia and neutrophil storage pool depletion are prominent features of experimental type II group B streptococci infection in neonates.
\end{abstract}

\section{Speculation}

Although neutrophil storage pool depletion is rare in infected adult animals or humans, its presence can be correlated with an increased mortality. It appears that neutrophil storage pool depletion is a prominent feature of experimental neonatal type II group $B$ streptococci infection. The resulting deficiency of phagocytic cell supply probably represents diminution in the already compromised host defense mechanism of the neonate. Thus, it is postulated that neutrophil storage pool depletion represents a pathophysiologic factor contributing to the high mortality rate observed in neonatal type II group B streptococci infection.

We recently have observed that pronounced depletion of the neutrophil stores in bone marrow aspirates of infected human neonates correlated with a fatal outcome (2). This finding suggested that neutrophil storage pool (NSP) exhaustion, with attendant diminution in neutrophil supply to infected tissues may, in some instances, play a role in the neonate's unusual susceptibility to overwhelming septicemia (11). This hypothesis has led us to explore, in a quantitative fashion, the circulating and storage neutrophil changes in experimental neonatal sepsis.

Because we had observed NSP depletion in human neonates with type II group B streptococci (GBS) sepsis, we elected to simulate human GBS disease in an animal model and to observe the accompanying neutrophil changes. We therefore developed an animal system in which rats in the first $12 \mathrm{hr}$ of life underwent inhalation inoculation with GBS. These animals subsequently developed the early mortality and pathologic findings describs in the early onset form of human GBS disease $(4,8)$. We ha used this model to define the circulating and storage neutropt changes which occur with experimental GBS infection.

\section{MATERIALS AND METHODS}

PREPARATION OF GROUP B STREPTOCOCC

GBS from an infected infant was identified by the precipiti method using rabbit antisera (7) and was grown overnight at $37^{\circ}$ in Todd-Hewitt broth. Aliquots were frozen at $-70^{\circ} \mathrm{C}$. Prior administration to the animals, an aliquot was thawed, inoculate into fresh Todd-Hewitt broth, and grown to logarithmic phase ( to $5 \mathrm{hr}$. To detect possible bacterial contamination, an inoculu was plated onto a sheep blood agar plate. Bacteria were the sedimented by centrifugation, and the concentrated organisn were washed three times in sterile phosphate-buffered saline. standard suspension was prepared by diluting the concentrater washed organisms in phosphate-buffered saline to an optic: density of 0.9 at $620 \mathrm{~nm}$ (14). These suspensions contained af proximately $5 \times 10^{\text {ti }}$ colony-forming units $/ 5 \mu 1$.

\section{ANIMAL. INOCULATION}

Sprague Dawley rats (15) in the first $12 \mathrm{hr}$ of life were usec Bacterial suspensions in $5 \mu \mathrm{l}$ were instilled into the anterior nare with a glass syringe (16). The animals' mouths were held shu until the inoculum had been aspirated. Control animals receive $5 \mu \mathrm{l}$ of sterile PBS intranasally. Rats were then placed 10 to 12 cage with the mothers. Twelve-wk-old rats anesthetized witl methoxyfluorane $(17)$ received $1 \times 10^{6}$ organisms/g body wt is one $\mu \mathrm{l}$ phosphate-buffered saline per g body wt (the same dos which produced $50 \%$ mortality in neonatal rats).

\section{('ULTURES OF INOCULATED ANIMALS}

To detect systemic infection with group B streptococci, culture were performed on blood, lung, spleen, kidney, liver, and brain o animals inoculated with group B streptococci and also on contro animals inoculated with PBS. Blood samples were inoculate directly onto sheep blood agar plates and into Todd-Hewitt broth Organ specimens were homogenized with $1 \mathrm{ml}$ Todd-Hewitt broth plated directly onto sheep blood agar plates, and also grown ir Todd-Hewitt broth overnight.

\section{QUANTITATIVE STUDIES OF CIRCULATING; AND STORAGF NEUTROPHILS}

Nucleated blood cell counts were performed electronically (18 in triplicate. and the average was determined. Cover slip blooc smears were stained with Wright's stain and subjected to a 200 . cell differential count. The mature NSP [all polymorphonuclear: 
'MN's), bands, and metamyelocytes] were measured from the ver, spleen, and femoral marrow in the following manner. The ver and spleen were removed and separately sieved in 1-cc lanks' balanced salt solution through a steel mesh gauze with 700 ores $/ \mathrm{cm}^{2}$. Then the suspensions were counted electronically in iplicate, and the average was recorded. Wright's stained smears f the suspensions were prepared and subjected to a 500- 10 1000ell differential count.

To obtain bone marrow specimens, the femurs were removed, nd a 25 gauge needle was inserted into the femoral head through hich $1.0 \mathrm{ml}$ Hanks' balanced salt solution was flushed with a tberculin syringe (1). The resulting cell suspensions were counted lectronically, and a 1000-cell differential count was performed.

\section{RESULTS}

\section{ANIMAL. INOCULATION MORTALITY STUDIES}

Forty-eight newborn rats weighing 4.5 to $5.5 \mathrm{~g}$ experienced $56 \%$ cortality after intranasally receiving $1 \times 10^{6} \mathrm{GBS}$ organisms per body wt. Eighty-five percent of all deaths occurred between 24 nd $48 \mathrm{hr}$ after inoculation with an occasional animal dying as ate as $1 \mathrm{wk}$ postinoculum. Postmortem studies included bacterial ultures of the brain, lungs, liver, spleen. kidneys, and blood which vere all positive for GBS. Hematoxylin- and eosin-stained postnortem tissue sections confirmed the presence of pneumonia and
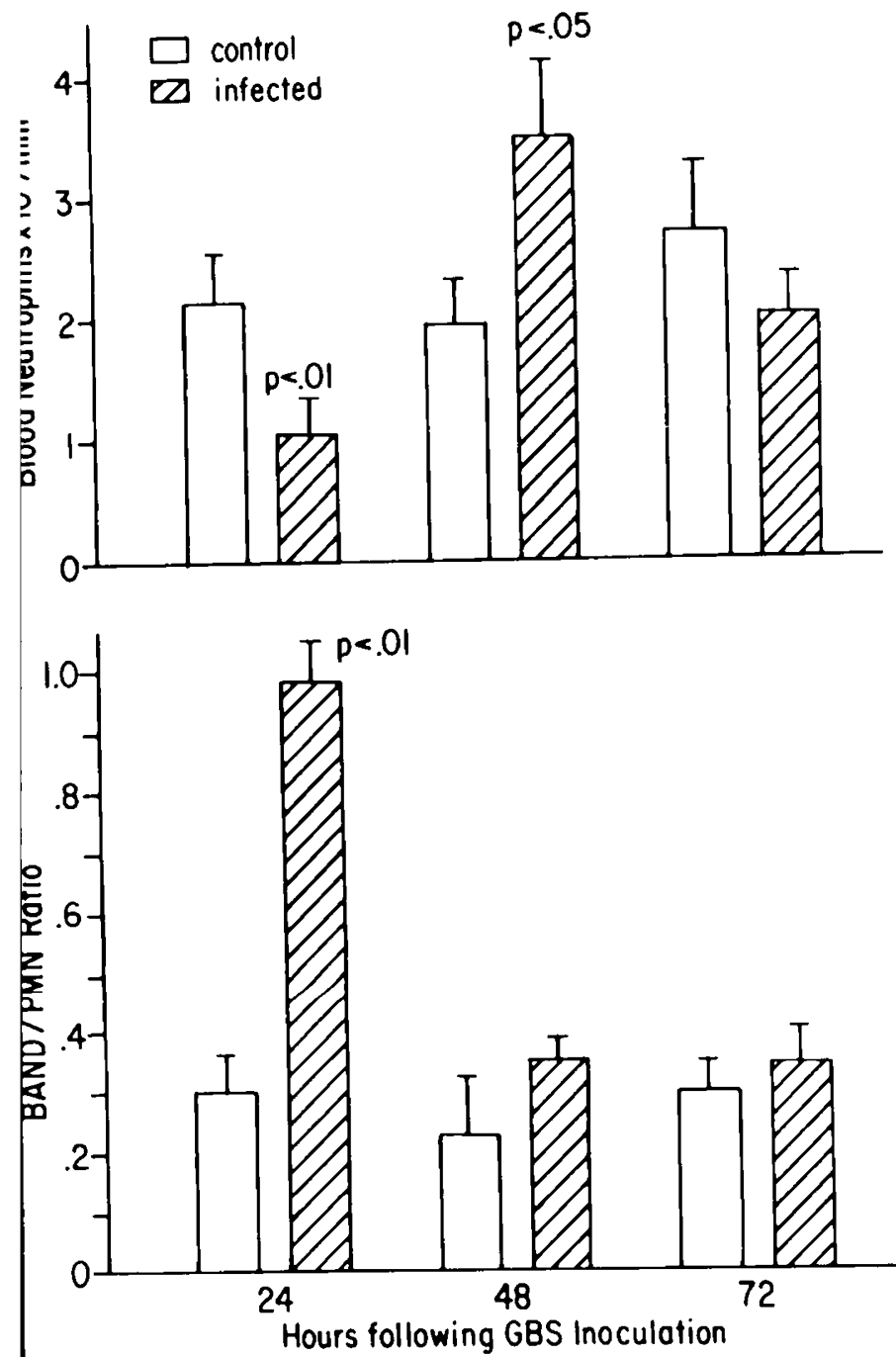

Fig. 1. a. blood neutrophil count in groups of infected and control rats $24 \mathrm{hr}$ following inoculation and in survivors 48 and $72 \mathrm{hr}$ following inoculation. Bracket, S.D.; $b$, band/PMN ratio in groups of infected and control rats following inoculation. Bracket, S.D.
NSP Femur
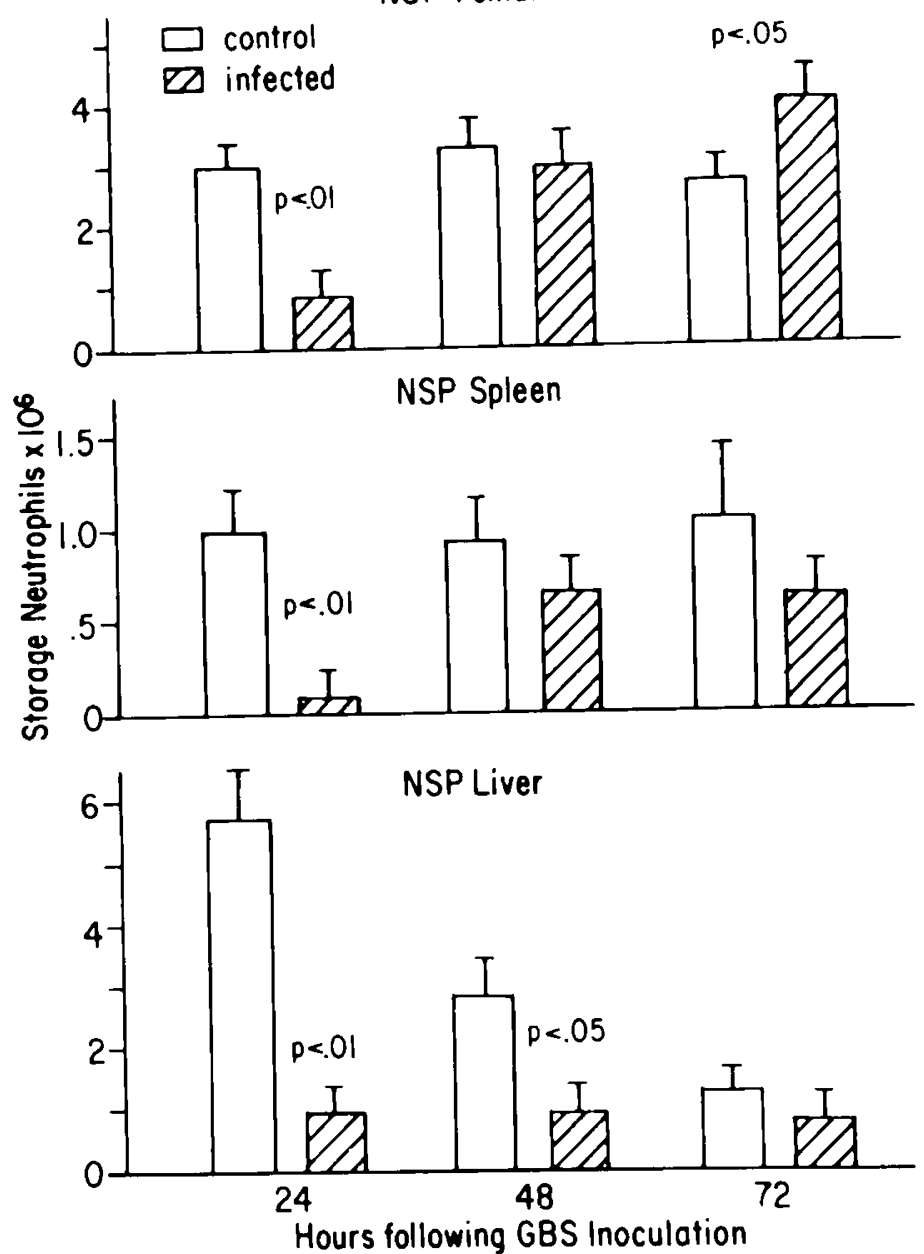

Fig. 2. a, mature neutrophils in the femur in groups of infected and control rats 24,48 , and 72 hr following inoculation; $h$, mature neutrophils in the spleen in both groups following inoculation: $c$, mature neutrophils in the liver in both groups following inoculation. Bracket, S.D.

demonstrated areas of pulmonary macro- and microhemorrhage. Hepatic and splenic congestion were seen, but no pathological changes were observed in the brain or meninges. In 10 twelve-wkold rats. the same dose of organisms per g body wt produced no positive blood cultures, no mortality, and no recognizable morbidity. In 28 neonatal rats receiving a much lower intranasal inoculation of $2 \times 10^{1} \mathrm{GBS}, 2$ deaths (7\% mortality) were observed. Opsonic antibody specific for GBS were not found in any of the sera of either the 12 -wk-old or the neonatal rats $(3)$.

\section{QUANTITATIVE STUDIES OF CIRCULATINC; AND STORAC;} NEUTROPHILS

Iwenty-four hi lollowing intrandsal GBS incoulation (a time when no mortality had yet occurred), neutropenia (Fig. la) and an elevated band $/$ PMN ratio (Fig. $1 b$ ) were detected $(P<0.01)$. NSP assessment showed a reduction of the mature neutrophils (PMN, bands, and metamyelocytes) in femoral splenic and hepatic storage compartments $(P<0.01)$ but no reduction in the proliferative (myeloblast, promyelocyte, and myelocyte) neutrophil pool (Fig. 2).

Between 24 and $48 \mathrm{hr}$ following inoculation. $85 \%$ of the GBS recipients died. Those surviving to $48 \mathrm{hr}$ were seen to have neutrophilia (Fig. $1 a$ ) with band/PMN ratios and NSP measurements which did not differ from controls. Those animals surviving $72 \mathrm{hr}$ showed no difference in neutrophil count. PMN ratio, or splenic or hepatic NSP size. They did, however, have an elevation of storage neutrophils in the femur (Fig. 2) compared to controls. 


\section{DISCUSSION}

A decrease in the number of circulating neutrophils has previously been cited as a poor prognostic factor in neonatal bacterial infection $(5,13)$. In the present study, we have attempted to determine whether bacterial infection in neonatal rats is associated with neutropenia and whether this neutropenia was associated with a decrease in the number of neutrophils stored in the marrow spleen or liver. Animals were inoculated intranasally with GBS to utilize a common human pathogen and to simulate a likely portal of entry in human GBS infection (4). Clinical infection with GBS did ensue, and postmortem study of the animals who died revealed GBS infection including pneumonia and sepsis. Interestingly, adult animals resisted GBS infection in a manner similar to that of adult humans (3). In infected neonatal rats, the mean blood neutrophil count was only $54 \%$ of the control value $(P<0.01)$, and coincident with this neutropenia, a reduction in the NSP was observed with similar depletion of marrow, liver, and splenic sites.

The process leading to exhaustion of the marrow NSP appeared to spare the neutrophils capable of proliferation, inasmuch as the number of myeloblasts, promyelocytes. and myelocytes was unaltered by GBS infection. It might be postulated, therefore, that the exhaustion of mature neutrophil supply occurred as a result of extensive mobilization of stored neutrophils from the marrow. Alternatively, a selective intramyeloid destruction of mature neutrophils may have occurred. In either case, the result would be the same, an inadequate supply of neutrophils available for mobilization and delivery to infected sites. In the neonatal rat (and in the premature human as well), neutrophil stores exist both in the marrow cavities and in the liver and spleen (12). In the present model, we demonstrate that all sites of neutrophil stores are available for mobilization in infection because depletion of bone marrow, liver, and splenic fractions occurred in a similar fashion. Also of note is that repletion of the bone marrow neutrophil stores took place, although repletion of the liver and splenic pools did not. This observation is in keeping with the limitation of myelopoiesis to the bone marrow in developing fetuses and neonates (6), as well as with our own noninfected rats during the first three days of life. It is of note that in the present model of bacterial infection, neutropenia reflected a depletion of neutrophil stores. such depletion may be of importance in reducing host resistance to bacterial invasion because in this state, inadequate numbers of neutrophils are available for mobilization to infected tissues. Indeed, the temporal relationship of the NSP depletion, neutropenia, and mortality suggest that NSP depletion contributed to the mortality. However, this hypothesis remains to be tested directly. We have also observed NSP depletion during bacterial infection in human neonates, both at autopsy and in four living neonates who subsequently died of bacterial infection (2). Other experimental animal evidence of NSP exhaustion leading to neutropenia and death can be found in the studies of Marsh et al. (9), who correlated mortality with NSP exhaustion in adult dogs infected with pneumococci. In adult humans with systemic ba terial infection, however, neutropenia secondary to NSP exhau tion is distinctly uncommon. It is occasionally seen in compr mised hosts such as chronic alcoholics and in this context regarded as a grave prognostic indicator $(10)$. In certain respec1 the human neonates seem to be a compromised host. They a unusually susceptible to bacterial infection, and often great diff culty is encountered attempting to sterilize a bacterial proce despite adequate antibiotic treatment (11). Perhaps contributir to this condition is the phenomenon which we observed in the: infected neonatal rats, diminution in the mature neutrophil stor in the marrow, spleen. and liver, with accompaning neutropen and subsequently diminished neutrophil delivery to the infecte tissues.

\section{REFERENCES AND NOTES}

1. Chervenick, P. A.. Boggs, D. R., Marsh. J. ( .. Cartwright. (;. E.. and Wintrob M. M.: Quantitative studies of blood and bone marrow neutrophils in norm mice. Am. J. Physiol., 2.5: 353 (1968)

2. Christensen. R. D.. and Rothstein. (i.: Exhaustion of mature marrou neutrophi in neonates with sepsis. J. Pediatr.. 96: $316(1980)$.

3. Hemming. V. G.. Hall. R. T., Rhodes, P. ( ., Shigeoka. A. O.. and Hill. H. R Assessment of group B streptococcal opsonins in human and rabbit serum $t$ neutrophil chemiluminescence. J. Clin. Invest.. 5.8: 1379 (1976).

4. Hemming. V. (;.. McCloskey. D. W.. and Hill. H. R.: Pneumonia in the neonat associated with group B streptococial septicemia. Am. J. Dis. Child., 130): 123
(1976).

5. Hutter. J. J.. Hathaway. W. E., and Wayne, E. R.: Hematologic abnormalities i severe neonatal necrotizing enterocolitis. J. Pediatr.. \&\&: $1(026$ (1976).

6. Knoll. W.: Die Blutbildung biem Embryo. Acta Haematol., 2: 369 (1949).

7. Lancefield. R. ( $\therefore$ A micro-precipitin-technique for classifying hemolytic stref tococit and improved methods for producing antisera. Proc. Soc. Exp. Bio Med.. 38: 473 (1938)

x. Manroe. B. I... Rosenfeld. (. R.. Weinberg. A. C;., and Browne, R.: Th differential leukocyte count in the assessment and outcome of early onse neonatal group B streptococal disease: J. Pediatr. 9/: 6.32 (1977).

9. Marsh. J. (.. Boggs, D. R.. Cartwright. G. E.. and Wintrobe. M. M.: Neutroph kinetics in acute infection. J. Clin. Invest., 40: 1943 (1967)

10. McFarland. W.. and Libre. E. P.: Abnormal leukocyte reponse in alcoholism Ann. Intern. Med., 59: 865 (1963)

11. Miller. M. E.: Host Defenses in the Human Neonate. In: Monographs it Neonatology. p. I (Grune \& Stratton. Inc.. New York. 1978) 12. Wintrobe. M. M.: Clinical Hematology. p. 55 (Lea \& Febiger. Philadelphia
1976).

13. Xanthou. M.. Leukecyte blood picture in ill newborn babies. Arch. I)s. (hild 47: 741 (1972).

14. Spectronic 20: Bausch and Lomb. Inc.. Rochester, NY

15. Simonson Laboratories, (iilroy, (A.

16. Hamilton Co. Whittier. CA

17. Abboll Laboratories. North Chicago, IL.

18. Coulter Electronics, Hialea. FL.

19. The authors thank John W. Athens, M.D. for his critical review of the manuscrip and Jane Macfarlane for her technical assistance.

20. Requests for reprints should be addressed to: Dr. R. D). (hristensen. Division o Hematology, 50 North Medical Drive. Salt Lake (ity. UT 84132 (USA).

21. This research was supported in part by United States Public Health Servic grants AI 13150) and AM21140 and a grant from the Thrasher Foundation 22. Received for publication July II, 1979

23. Accepted for publication August 30, 1979. 